\title{
Aging and the Neural Correlates of Successful Picture Encoding: Frontal Activations Compensate for Decreased Medial-Temporal Activity
}

\author{
Angela H. Gutchess ${ }^{1}$, Robert C. Welsh' ${ }^{1}$, Trey Hedden ${ }^{2}$, \\ Ashley Bangert ${ }^{1}$, Meredith Minear ${ }^{1}$, Linda L. Liu ${ }^{1}$, and Denise C. Park ${ }^{3}$
}

\begin{abstract}
We investigated the hypothesis that increased prefrontal activations in older adults are compensatory for decreases in medial-temporal activations that occur with age. Because scene encoding engages both hippocampal and prefrontal sites, we examined incidental encoding of scenes by 14 young and 13 older adults in a subsequent memory paradigm using functional magnetic resonance imaging (fMRI). Behavioral results indicated that there were equivalent numbers of remembered and forgotten items, which did not vary as a function of age. In an fMRI analysis subtracting forgotten items from remembered items, younger and older adults both activated inferior frontal and lateral occipital regions bilat-
\end{abstract}

\section{INTRODUCTION}

The present study focuses on age differences in neural activations associated with the encoding of complex scenes. Scenes elicit a high degree of activity in both frontal and medial-temporal regions of the brain, and for this reason, represent an important medium for understanding age differences in neural function. Although verbal materials typically activate left frontal areas at encoding, picture encoding paradigms often activate both hemispheres in frontal areas (Golby et al., 2001; Kelley et al., 1998), reflecting increased encoding requirements for pictures, or the dual coding of pictures in visual and verbal formats (Kelley et al., 1998; Paivio \& Csapo, 1973). In addition to increased frontal activation, pictures, particularly complex scenes, strongly activate hippocampal and parahippocampal structures (Kelley et al., 1998; Henke, Buck, Weber, \& Wieser, 1997; Stern et al., 1996). Evidence suggests that the robust medialtemporal activations reflect relational processing, that is, the binding or integration of disparate perceptual elements into a coherent representation (Henke, Weber, Kneifel, Wieser, \& Buck, 1999; Henke, Buck, et al., 1997),

\footnotetext{
${ }^{1}$ University of Michigan, ${ }^{2}$ Stanford University, ${ }^{3}$ University of Illinois
}

erally; however, older adults showed less activation than young adults in the left and right parahippocampus and more activation than young adults in the middle frontal cortex. Moreover, correlations between inferior frontal and parahippocampal activity were significantly negative for old but not young, suggesting that those older adults who showed the least engagement of the parahippocampus activated inferior frontal areas the most. Because the analyses included only the unique activations associated with remembered items, these data suggest that prefrontal regions could serve a compensatory role for declines in medial-temporal activations with age.

which complex stimuli such as pictures induce (Cohen et al., 1999; Stern et al., 1996).

In a study investigating encoding of complex scenes as a function of age, younger adults showed greater hippocampal activations than older adults while encoding the scenes, whereas older adults showed more activity in left and right inferior frontal regions (Park, Welsh, et al., 2003). Park and Gutchess (in press) and Park, Welsh, et al. (2003) speculated that the additional prefrontal activations seen in older adults were compensatory for the decreased hippocampal activations older adults evidenced, but their study design did not permit a linkage of these activations to successful encoding. In the present study, we investigate the hypothesis that increased frontal activations in older adults are linked to low activity in medial-temporal areas using a subsequent memory paradigm that permits investigation of items that are successfully encoded in contrast to those that are forgotten. The finding of decreased medial-temporal involvement for older adults compared to young adults during encoding is robust and has been reported in a number of studies, including encoding of faces (Grady, McIntosh, Horwitz, et al., 1995), natural scenes (Park, Welsh, et al., 2003), verbal materials (Daselaar, Veltman, Rombouts, Raaijmakers, \& Jonker, 2003; Grady, McIntosh, Rajeh, Beig, \& Craik, 1999), and line drawings 
(Grady, McIntosh, Rajah, Beig, et al., 1999). Generally, when verbal materials are used, hippocampal activations are more likely to show age equivalence, particularly under incidental encoding conditions (Daselaar, Veltman, Rombouts, Lazeron, et al., 2003; Morcom, Good, Frackowiak, \& Rugg, 2003). The only long-term memory studies in which the elderly have exhibited greater hippocampal or parahippocampal activations than the young are studies involving retrieval. In one case, greater hippocampal activations occurred in older adults when they retrieved words presented a few minutes earlier (Cabeza, Daselaar, et al., 2004), whereas in another study, older adults showed more hippocampal activations when they retrieved autobiographical memories (Maguire \& Frith, 2003).

Age patterns in frontal regions in long-term memory studies are more complex than those reported for medial-temporal regions. A primary focus of the aging and neuroimaging literature has been on understanding patterns of increased bilaterality with age in frontal areas where young adults tend to be lateralized (Cabeza, 2002; Reuter-Lorenz, 2002; Reuter-Lorenz et al., 2001). Bilaterality in older adults is most commonly manifested when young adults evidence unilateral activation in a single hemisphere for a task, and older adults activate this hemisphere as well as the homologous region of the contralateral hemisphere (Cabeza, Anderson, Locantore, \& McIntosh, 2002; Grady, Bernstein, Beig, \& Siegenthaler, 2002; Rosen et al., 2002; Reuter-Lorenz, Jonides, et al., 2000; Madden et al., 1999; Bäckman et al., 1997; Cabeza, Grady, et al., 1997). In addition to the typical bilaterality pattern resulting from high levels of activation in both hemispheres for older adults, a second pattern of bilaterality sometimes occurs. This second case results when young adults strongly activate a single hemisphere, whereas older adults show reduced activation in this hemisphere along with equivalently low levels of activation in the contralateral hemisphere (Logan, Sanders, Snyder, Morris, \& Buckner, 2002; Stebbins et al., 2002). Bilateral activation patterns in old but not young have been reported for working memory tasks (Reuter-Lorenz, Jonides, et al., 2000), incidental memory encoding (Logan et al., 2002; Rosen et al., 2002), and retrieval tasks (Cabeza, Anderson, et al., 2002; Grady, Bernstein, et al., 2002; Madden et al., 1999; Bäckman, et al., 1997; Cabeza, Grady, et al., 1997).

Cabeza (2002) and Reuter-Lorenz (2002) have argued that in tasks where young show unilateral activation, bilateral hemispheric activation in older adults indicates compensation for a declining neural system. The strongest evidence to date in favor of the compensation argument comes from comparisons between high- and low-performing elderly. Using verbal memory tasks, Cabeza, Anderson, et al. (2002) and Rosen et al. (2002) both found that high performers activated prefrontal sites bilaterally, whereas low-performing elderly and young exhibited unilateral activations. Reuter-Lorenz,
Jonides, et al. (2000) found that faster elderly showed more bilaterality than slower elderly. Other cases, however, suggest that additional activations may be nonfunctional. Logan et al. (2002) found that bilaterality was more characteristic of the oldest participants relative to younger elderly, suggesting that bilaterality was not related to superior performance. Likewise, heightened activation was more characteristic of dysfunctional aging in Lustig et al.'s (2003) study comparing Alzheimer's patients, normal elderly adults, and young adults.

Although comparisons of individual differences help to clarify the role of patterns of activations across the lifespan (Hedden \& Gabrieli, 2004), within-subject comparisons of activations associated with successful versus unsuccessful encoding are also an important means for assessing whether increased neural activations in older adults are functionally related to task performance. In the subsequent memory paradigm (Brewer, Zhao, Desmond, Glover, \& Gabrieli, 1998; Wagner, Schacter, et al., 1998), an event-related design is used to isolate successful encoding trials from unsuccessful encoding trials by examining responses on a recognition test to determine which encoded items were later remembered. Thus, it is possible to examine activation patterns associated with correctly remembered items and determine how they differ from forgotten items. If additional activation in frontal areas is functional in older adults, one might expect to see more activity for remembered compared to forgotten items. Because past subsequent memory studies have suggested that increased engagement of both frontal and medial-temporal regions is related to the likelihood of later memory for an item (Daselaar, Veltman, Rombouts, Lazeron, et al., 2003; Morcom et al., 2003; Kirchhoff, Wagner, Maril, \& Stern, 2000; Brewer et al., 1998; Wagner, Schacter et al., 1998), it is an ideal paradigm for understanding age-related changes in frontal-hippocampal circuitry. In young adults, evidence from subsequent memory studies reveals a network in which prefrontal regions govern strategic and control processes that influence how medial-temporal regions accomplish long-term memory consolidation (Kirchhoff et al., 2000; Wagner, Schacter, et al., 1998).

To date, there have been two published reports on aging and subsequent verbal memory. The data converge to suggest that overall, similar networks discriminate successfully encoded items from unsuccessful ones in younger adults and older adults. Although older adults exhibited medial-temporal activations equivalent to younger adults for remembered items in Morcom et al.'s (2003) study, Daselaar, Veltman, Rombouts, Lazeron, et al. (2003) reported reduced perirhinal/parahippocampal involvement during encoding for older adults who performed poorly on a behavioral measure of recognition memory. Older adults with higher recognition performance showed medial-temporal activations similar to younger adults. Prefrontal sites also evidenced similar activation between old and young in these two 
studies, although Morcom et al. found evidence for increased bilaterality in old when prefrontal regions of interest (ROIs) were examined. The overall absence of age differences in frontal activations may be a result of the incidental encoding instructions used in these studies; elderly adults are most likely to show underrecruitment of frontal regions under intentional conditions (Logan et al., 2002).

Despite the finding of overall similar involvement with age of prefrontal and medial-temporal regions in successful encoding of verbal materials (Daselaar, Veltman, Rombouts, Lazeron, et al., 2003; Morcom et al., 2003), a different pattern might be expected for the encoding of complex scenes. Because picture encoding results in higher activations than verbal stimuli in both medialtemporal (Reber, Wong, \& Buxton, 2002; Kirchhoff et al., 2000; Grady, McIntosh, Rajah, \& Craik, 1998) and bilateral prefrontal areas (Golby et al., 2001; Kelley et al., 1998), we hypothesized that under conditions of such high neural load, age differences in frontal and medialtemporal engagement would become apparent for remembered compared to forgotten items. In line with the hypothesis that older adults show increased prefrontal activation to compensate for deficient hippocampal activations, we expected that young adults would show greater medial-temporal activations than older adults for remembered compared to forgotten items. In contrast, we expected that older adults would show increased compensatory prefrontal activation when successfully encoding scenes compared to younger adults. Because encoding of pictorial stimuli engages bilateral prefrontal regions in younger adults (Golby et al., 2001; Kelley et al., 1998), the common finding of increased bilaterality with age was unlikely to occur. Thus, rather than predicting a pattern of bilaterality in older adults and unilaterality in younger adults, we hypothesized that older adults would show increased prefrontal activations in unique regions not activated by young adults. There is some evidence for unique activation of prefrontal areas by older adults in nonhomologous regions. For example, the elderly activated more dorsolateral prefrontal and medialtemporal areas on a visual gradient working memory task (McIntosh et al., 1999) and showed increased activation in the left and right inferior frontal regions in a scene working memory task (Park, Welsh, et al., 2003) compared to young adults. Inferior frontal activations have been routinely reported for younger adults in subsequent memory studies (Brewer et al., 1998; Wagner, Schacter, et al., 1998); therefore, we predicted that in addition to activating inferior frontal sites, older adults would also activate the dorsolateral prefrontal cortex as a unique region. This would be consistent with reports of additional dorsolateral activation for older adults on difficult tasks (Rypma \& D'Esposito, 2000), in light of age-impaired automatic encoding that relies on medialtemporal structures (Grady, McIntosh, \& Craik, 2003; Moscovitch \& Winocur, 1992). Of particular importance is that these predictions involve activity related to remembered items compared to forgotten items. Thus, any areas of increased prefrontal activation associated with remembered items in the elderly are almost certainly functional, that is, the activation of the area above the level of the young enhances task performance for the old. In the present article, we will use the term "compensation" to describe neural resources activated above the level of young adults that are in the service of successful task performance. It is important to note that this definition suggests that increased activation of functional (compensatory) neural resources can occur during both incidental and intentional encoding tasks, as the definition is based on the functionality of activations rather than active attempts to recruit neural resources.

In the present study, we investigated potential age differences in the correlates of subsequent memory for pictures of complex scenes. Fourteen young and 13 elderly adults incidentally encoded full-color photographs of scenes for $4 \mathrm{sec}$ each. After a 10-min delay, they received a surprise recognition test with the original photographs and highly similar lures (Figure 1) and indicated with a keypress whether they remembered the item with high confidence, remembered the item with low confidence, or identified it as a new item. Based on recognition performance, the encoding trials were sorted as to whether or not the picture was later remembered ("remembered-high confidence" response) or forgotten ("no, did not study" response). In past subsequent memory studies, there have been difficulties in acquiring sufficient numbers of "forgotten"
Figure 1. Example of an encoded picture and a recognition lure matched for perceptual similarity and semantic content.

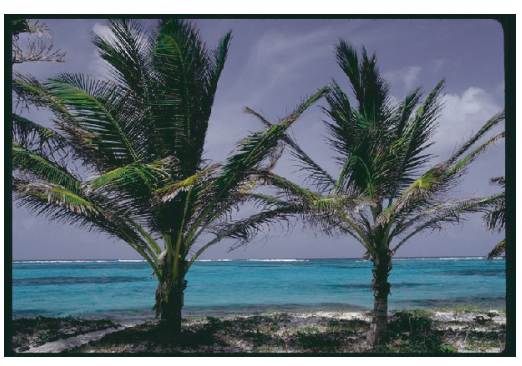

Encoded picture

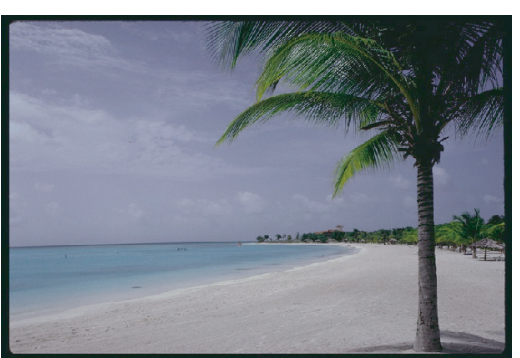

Recognition lure 
items (e.g., "misses" where a subject says a presented item was not studied) to have sufficient power to make contrasts between remembered and forgotten items (Daselaar, Veltman, Rombouts, Lazeron, et al., 2003; Daselaar, Veltman, Rombouts, Raaijmakers, \& Jonker, 2003). In the present study, photographs of outdoor scenes were used as targets and based on past work, recognition lures were created that resulted in equivalent numbers of remembered and forgotten items for both age groups (Park, Welsh, et al., 2003; Smith, Park, Cherry, \& Berkovsky, 1990; Park, Puglisi, Smith, \& Dudley, 1987; Park, Puglisi, \& Smith, 1986) while simultaneously avoiding very low "miss" rates. This resulted in sufficient power to subtract forgotten from remembered items for both old and young adults.

\section{RESULTS}

\section{Behavioral Data}

We discarded low confidence responses and used only high confidence responses, consistent with past subsequent memory studies (Wagner, Schacter, et al., 1998). As in other studies, low confidence responses did not discriminate studied items from lures, with younger adults averaging an $A^{\prime}$ of .50 and older adults averaging an $A^{\prime}$ of .49 in our data (an $A^{\prime}$ of .5 represents chance performance, whereas a score of 1 indicates perfect performance; Snodgrass \& Corwin, 1988). To test if there were equivalent numbers of hits and misses for a subsequent memory analysis, we conducted a mixed analysis of variance with age (young/old) as a betweensubjects variable and response type (hits/remembered or misses/forgotten) as a within-subject variable. Means are presented in Table 1 . There were no significant main effects of age $[F(1,25)=.19, p>.65]$ or response type $[F(1,25)=.61, p>.40]$ and the interaction of the two was also nonsignificant $[F(1,25)=.24, p>.60]$. This suggested that we succeeded in matching the number of remembered and forgotten responses, avoiding the problems some past studies of word memory have faced with insufficient power due to too few forgotten responses (Daselaar, Veltman, Rombouts, Lazeron, et al., 2003; Kirchhoff et al., 2000). We also examined age differences for false alarms to new lures tested at recognition. Older adults made somewhat more false alarms than younger adults $[t(25)=2.47, p<.03]$, which led to an advantage for younger adults on $A^{\prime}$ scores, as they are calculated using hit rates and false alarm rates $[t(25)=2.83, p<.01]$.

\section{Imaging Data}

\section{Common Regions Associated with Remembered Items}

As an initial step, we examined shared areas of activation across young and elderly participants for remembered compared to forgotten items. The subtraction showed bilateral inferior frontal activations (BA 45/46) common to younger and older adults. This is consistent with past studies that reported common inferior frontal activations (although those were mostly left-lateralized in response to verbal materials; Daselaar, Veltman, Rombouts, Lazeron, et al., 2003; Morcom et al., 2003). Second, in contrast to previous studies, there were no common activations in the hippocampus and surrounding regions. Finally, we noted large common areas of activation extending from secondary visual areas into the dorsal and ventral streams, most notably fusiform regions, consistent with past reports of activation of higher-order visual processing areas (Daselaar, Veltman, Rombouts, Lazeron, et al., 2003; Morcom et al., 2003; Kirchhoff et al., 2000). Results of these analyses appear in Figure 2 and details are available in Table 2.

\section{Regions Uniquely Associated with Remembering as a Function of Age}

Next, we assessed areas uniquely activated by younger or older adults for remembered items. Compared to the elderly, the young demonstrated additional activations centered bilaterally in the parahippocampal gyrus and extending into the fusiform gyrus (see Figure $3 \mathrm{~A}$ ), as well as lateral occipital gyri activations. These activations are consistent with past subsequent memory effects in parahippocampal regions for young adults in studies of pictures and words (Kirchhoff et al., 2000; Brewer et al., 1998; Wagner, Schacter, et al., 1998). Older adults showed increased activation for remembered items bilaterally in the dorsolateral (middle frontal, BA 8) cortex, but primarily on the left (Figure 3B). A similar region was noted in Morcom et al.'s (2003) study of verbal memory in the combined analysis of younger and older adults and work by Stebbins et al. (2002) showed the region had a different relationship to verbal memory with age, suggesting that the region could be involved in success-

Table 1. Performance on Memory Task by Age (Means and Standard Deviations)

\begin{tabular}{lcccc}
\hline & $\begin{array}{c}\text { Remembered Items } \\
\text { (High Confidence Hits) }\end{array}$ & $\begin{array}{c}\text { Forgotten Items } \\
\text { (Misses) }\end{array}$ & $\begin{array}{c}\text { False Alarms } \\
\text { (High Confidence) }\end{array}$ & $\begin{array}{c}A^{\prime} \text { Score (High Confidence Hits } \\
\text { and False Alarms) }\end{array}$ \\
\hline Young & $0.39(0.13)$ & $0.38(0.16)$ & $0.16(0.12)$ & $0.73(0.06)$ \\
Old & $0.43(0.17)$ & $0.36(0.17)$ & $0.29(0.16)$ & $0.63(0.10)$ \\
Significance $(p)$ & 0.52 & 0.80 & 0.02 & 0.009 \\
\hline
\end{tabular}




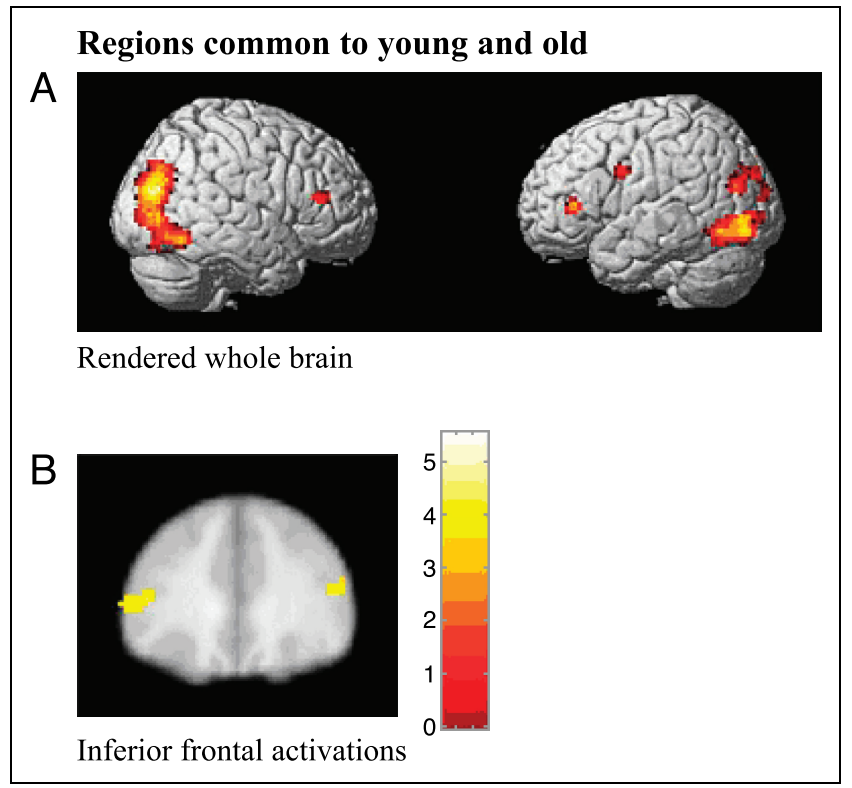

Figure 2. Regions of activation common to younger and older adults for the remembered minus forgotten contrast are rendered on a whole brain (A). The bilateral inferior frontal activations are highlighted in B, displayed on the average of the 27 participants' brains.

ful verbal encoding. However, this area may be used more heavily by the elderly during nonverbal encoding, as indicated by our data. The finding is also consistent with reports of an increased role in memory for the dorsolateral prefrontal cortex with age (Grady, McIntosh, \& Craik, 2003). Older adults also showed more activation than younger adults in the left parietal region, specifically the supramarginal gyrus, which was also identified as a region activated more by old than young in the Morcom et al. study. A large area of activation in the old also occurred in the medial frontal cortex in the region of the anterior cingulate, extending from BA 32 into BA 10 (Figure 3C). This age difference, however, largely reflects more activation for forgotten items than remembered items by younger adults, a pattern that is absent in older adults. Because this region was more active for forgotten items than remembered items, it did not represent a subsequent memory effect for the old but rather a subsequent forgetting effect for the young. Our subsequent forgetting region is in the vicinity of the effect seen in the young in Morcom et al.'s study, although their activations were less medial (bilateral in BA 10) and did not extend into the anterior cingulate (see Table 3 for additional details on areas revealing age differences).

\section{Laterality Analysis}

Due to the considerable interest in changes to laterality with age (Cabeza, 2002; Reuter-Lorenz, 2002) and the finding of increased prefrontal bilaterality with age in a verbal subsequent memory paradigm (Morcom et al.,
2003), we queried the prefrontal and medial-temporal regions identified by our study for age differences in laterality. We found that there were no significant laterality differences with age for the inferior frontal regions common to both younger and older adults $[t(25)=1.34$, $p>$.19], the parahippocampal region activated more by younger adults $[t(25)=1.07, p>.29]$, or the middle frontal region activated more by older adults $[t(25)=$ $.96, p>.34]$.

\section{Correlational Analyses}

To assess the hypothesis that increased prefrontal activation in the old is associated with decreased medialtemporal activations, we examined connectivity between middle frontal, inferior frontal, and parahippocampal regions using Pearson's correlations (two-tailed). We included the inferior frontal activations in the analysis to address the possibility that middle frontal regions activated by older adults were unique regions recruited in tandem with frontal sites common to both age groups. The correlations are shown in Table 4. Although young adults generally showed positive correlations between inferior frontal and medial-temporal activations (although only the right parahippocampal to the left inferior frontal was significant), older adults consistently showed significant negative relationships, indicating that lower parahippocampal activation was associated with higher inferior frontal engagement. These relationships are displayed in Figure 4. Moreover, left middle frontal activations may play a supplementary role for older adults because their left middle frontal activations were correlated with inferior frontal activations, a relationship not demonstrated by the young.

\section{DISCUSSION}

The findings from the present study provide substantive support for the hypothesis that prefrontal activations, specifically in the dorsolateral prefrontal cortex, compensate for decreased medial-temporal activations in older adults. The results indicated that when activations associated with remembered items were contrasted with activations for forgotten items, there were areas of common activation between old and young in the left and right inferior frontal cortex and large regions of the posterior cortex. Despite these commonalities, support was present for the hypothesis of decreased medialtemporal but increased prefrontal activation in older adults. Young adults showed more left and right parahippocampal engagement for remembered versus forgotten items than older adults, whereas older adults showed more bilateral middle frontal engagement than younger adults. Moreover, there was evidence for a significant negative relationship between activations in the inferior prefrontal cortex and the parahippocampus in older adults, suggesting that they engaged the pre- 
Table 2. Regions of Significant Activation Common to the Young and Elderly

\begin{tabular}{|c|c|c|c|c|c|c|c|}
\hline \multirow[b]{2}{*}{ Region of Activation } & \multirow[b]{2}{*}{ Hemisphere } & \multirow[b]{2}{*}{$B A$} & \multicolumn{3}{|c|}{ MNI Coordinates } & \multirow[b]{2}{*}{ Peak Z } & \multirow[b]{2}{*}{ No. of Voxels } \\
\hline & & & $x$ & $y$ & $z$ & & \\
\hline \multirow[t]{2}{*}{ Brainstem } & & N/A & -3 & -27 & -3 & 4.46 & 78 \\
\hline & & & 6 & -30 & -6 & 4.41 & \\
\hline \multirow[t]{2}{*}{ Parietal, angular gyrus } & $\mathrm{R}$ & 39 & 39 & -69 & 30 & 4.28 & 686 \\
\hline & & & 30 & -66 & 27 & 4.07 & \\
\hline Lateral occipital cortex & $\mathrm{R}$ & $37 / 19$ & 51 & -60 & -12 & 4.04 & \\
\hline \multirow[t]{2}{*}{ Angular gyrus } & $\mathrm{L}$ & $39 / 19$ & -30 & -75 & 24 & 4.21 & 127 \\
\hline & & & -24 & -75 & 36 & 3.46 & \\
\hline Lateral occipital cortex & $\mathrm{L}$ & 19 & -18 & -87 & 30 & 3.43 & \\
\hline Precentral gyrus & $\mathrm{L}$ & 6 & -45 & 0 & 30 & 4.02 & 27 \\
\hline \multirow[t]{3}{*}{ Lateral occipital cortex } & $\mathrm{L}$ & 18 & -48 & -78 & -3 & 3.94 & 284 \\
\hline & & & -51 & -63 & -9 & 3.43 & \\
\hline & & & -36 & -90 & 0 & 3.30 & \\
\hline Superior parietal lobule & $\mathrm{L}$ & $7 / 40$ & -24 & -57 & 42 & 3.89 & 19 \\
\hline Inferior frontal & $\mathrm{L}$ & $45 / 46$ & -51 & 36 & 6 & 3.63 & 44 \\
\hline Inferior frontal & $\mathrm{R}$ & $45 / 46$ & 48 & 36 & 15 & 3.55 & 22 \\
\hline Lingual gyrus & $\mathrm{L}$ & $17 / 18$ & -6 & -60 & 3 & 3.45 & 14 \\
\hline Lateral occipital cortex & $\mathrm{L}$ & $19 / 18$ & -24 & -93 & 15 & 3.32 & 16 \\
\hline
\end{tabular}

Regions significant at an uncorrected $p<.001$ with an extent $>5$ for the remembered-high versus forgotten covariate.

A maximum of 3 local maxima at least $8 \mathrm{~mm}$ apart are displayed per region of activation.

$\mathrm{BA}=$ Brodmann area (approximate).

frontal cortex more when parahippocampal activations were low. Finally, in addition to the strong relationships between the parahippocampal and inferior frontal cortex, there was also evidence for additional left and right middle frontal activation in older adults when inferior frontal regions were activated. Because these relationships occurred for remembered items contrasted with forgotten items, they suggest functional compensation. A more detailed discussion of the results follows.

The finding of age-related under-recruitment of hippocampal or parahippocampal regions has not been reported in prior subsequent memory investigations of older adults with normal verbal memory performance (Daselaar, Veltman, Rombouts, Lazeron, et al., 2003; Morcom et al., 2003), although Cabeza, Daselaar, et al. (2004) reported increased parahippocampal activations in older adults during a verbal episodic retrieval task. However, because encoding of pictorial stimuli involves considerable relational processing supported by medial-temporal structures (Cohen et al., 1999), decreased parahippocampal engagement may occur primarily when tasks or stimuli place a heavy memory load on this region, analogous to findings that age-related differences in frontal activations occur under high but not low working memory loads (Rypma \& D'Esposito, 2000). Age-related decreases in activation of the hippocampus and surrounding regions are consistent with reports of volumetric declines in medial-temporal structures with age (as reviewed by Raz, 2000) that may impact function, and with reports from other studies investigating the processing of pictorial stimuli in older adults (Park, Welsh, et al., 2003; Grady, Bernstein, et al., 2002; Grady, McIntosh, Rajah, Beig, et al., 1999; Grady, McIntosh, Horwitz, et al., 1995).

Although decreased hippocampal and parahippocampal activations occur at encoding with age, we should note that there is some evidence that parahippocampal regions may activate more strongly for older adults under conditions of retrieval. In a verbal episodic retrieval task, the elderly showed more parahippocampal activations compared to the young, which Cabeza, Daselaar, et al. (2004) attributed to increased reliance on feelings of familiarity rather than explicit memory by older adults to make recognition judgments (Jennings \& Jacoby, 1993, 1997). Maguire and Frith (2003) reported a similar effect in the hippocampus for retrieval of auto- 


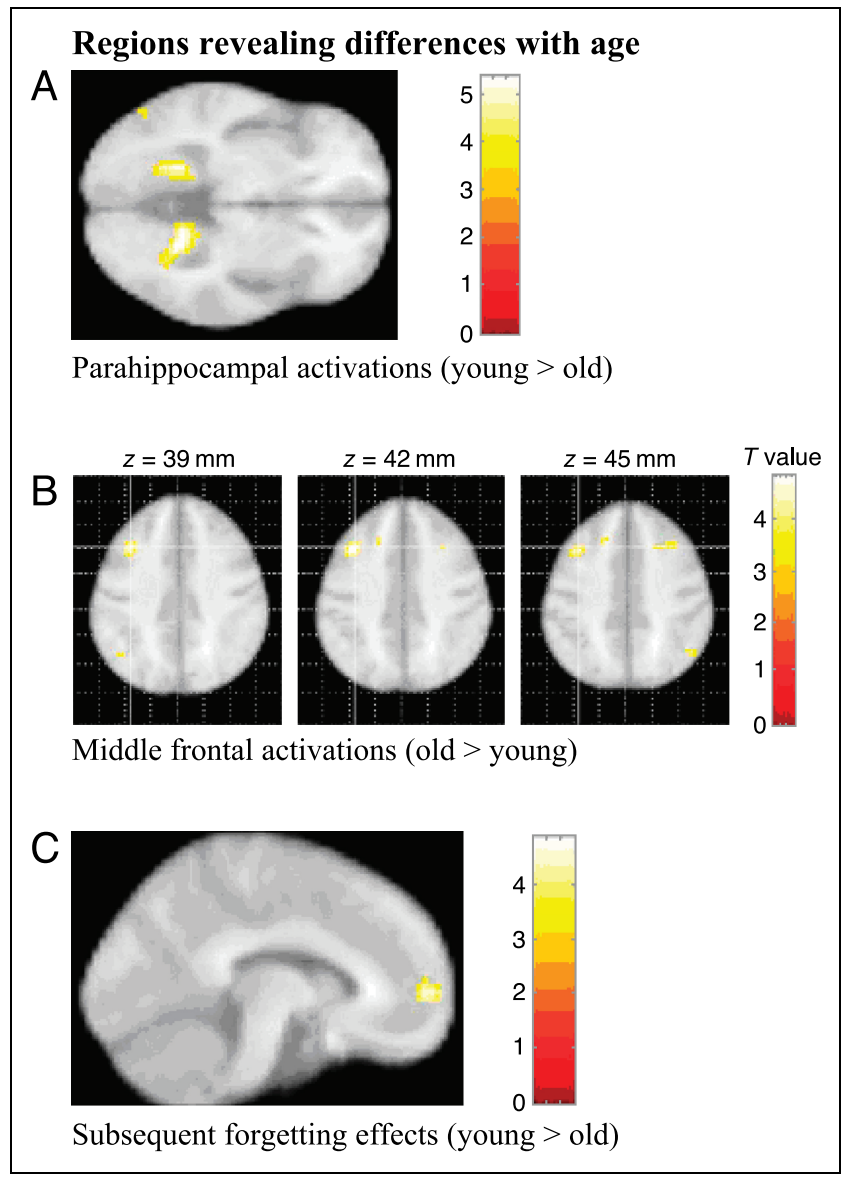

Figure 3. Age differences in subsequent memory (remembered minus forgotten contrast) are displayed on the average of the 27 participants' brains in $\mathrm{A}$ and $\mathrm{B}$, with younger adults activating more the bilateral parahippocampal cortex (A) and older adults activating more the middle frontal cortex (B). Subsequent forgetting effects (forgotten minus remembered contrast) are shown in (C) and reveal an age difference such that younger adults show larger subsequent forgetting effects in the frontopolar region than older adults.

biographical memories. They posited that the effect could reflect the additional search space for older adults to precisely localize the spatial or temporal context of the memory, drawing on Tulving's (2002) characterization of "mental time travel." The differences in parahippocampal and hippocampal activations between encoding and retrieval processes suggest that more precise parsing of anatomical localization of function may be fruitful, with different regions of the parahippocampus perhaps serving different functions and varying in susceptibility to age-related deterioration. In the present study, the parahippocampal activations are more posterior than those reported by Cabeza et al. and Maguire and Frith, supporting an anatomical separation that may be sensitive to different processes (e.g., familiarity vs. localization in time/space vs. relational processing). Although evidence is sparse for age effects in discrete parahippocampal regions that govern specific processes, dissociations have been identified for the hippocampus and the surrounding cortex in younger adults. Davachi, Mitchell, and Wagner (2003) suggested that the hippocampus and posterior parahippocampus contribute heavily to source recollection, consistent with a role in encoding associative relationships. In contrast, perirhinal regions support encoding and item recognition, which rely on large contributions from item familiarity. Further investigation of the processes that contribute to picture encoding will lead to better understanding of the function of specific medial-temporal subregions, as well as their vulnerability to age-related change.

With respect to frontal areas, the young and the elderly showed common activations in the bilateral inferior cortex, consistent with findings for encoding of pictures in young adults (Golby et al., 2001; Kelley et al., 1998). Additionally, however, we found that older adults activated middle frontal regions not activated by young adults. This activation of additional frontal regions is consistent with past work by Park, Welsh, et al. (2003) and McIntosh et al. (1999) who found additional activation in prefrontal sites that were not involved in the task for young, as well as functional connectivity analyses (Grady, McIntosh, \& Craik, 2003) indicating that the dorsolateral prefrontal cortex is more related to performance and hippocampal activations in older adults. The finding of increased prefrontal activation in Park et al. was accompanied by decreased hippocampal activations, but the pattern could not be isolated to successful encoding trials. Because in our data these activations were greater for encoding of items that were later remembered than for those items that were later forgotten, it suggests that this additional activation serves a compensatory function. Such compensation could occur through recruitment of additional processes to aid in task performance, consistent with theories of prefrontal cortex function (Miller \& Cohen, 2001) and reports of increased dorsolateral activations for difficult conditions (Rypma \& D'Esposito, 2000). The production-monitoring framework (Cabeza, Locantore, \& Anderson, 2003) proposes specific processes that could be increased by recruitment of the left and right prefrontal cortex, with the left hemisphere controlling semantic production and the right hemisphere governing monitoring and verification. Engagement of additional left and right regions by the elderly could reflect incidental attempts to draw on additional semantic or monitoring functions when faced with a demanding task such as encoding a large number of similar pictures.

The correlational data suggest that prefrontal activations in the elderly are compensatory for decreased engagement of medial-temporal areas. Because these correlations represent activations associated with remembered items contrasted with forgotten items, the correlations reflect neural activity specific to successful encoding. Older adults exhibited a strong negative relationship between parahippocampal and inferior 
$B A$

$\begin{array}{lll}x & y & z\end{array}$

Peak Z No. of voxels

$$
\text { Young > Elderly }
$$

Parahippocampal/fusiform gyrus

Parahippocampal/fusiform gyrus

Lateral occipital cortex

Lateral occipital cortex

Lateral occipital cortex

Elderly $>$ Young

Middle frontal gyrus

Anterior cingulate/Frontopolar

Precentral gyrus

Supramarginal gyrus

Lenticular nucleus

Supramarginal gyrus

Superior frontal

Caudate nucleus

Middle frontal gyrus/superior frontal gyrus

$\begin{array}{llrrrrr}\mathrm{R} & 37 / 36 & 24 & -48 & -3 & 4.33 & 146 \\ & & 33 & -57 & -6 & 3.79 & \\ \mathrm{~L} & 37 / 36 / 19 & -21 & -51 & -3 & 4.17 & 63 \\ & & -54 & -66 & -9 & 3.98 & 53 \\ \mathrm{~L} & 19 / 18 & -45 & -84 & -12 & 3.63 & 22 \\ \mathrm{R} & 19 & 54 & -75 & -9 & 3.31 & 10 \\ & & 57 & -63 & -12 & 3.17 & \\ \mathrm{R} & 19 & 45 & -84 & 15 & 3.30 & 10 \\ & & 48 & -81 & 6 & 3.17 & \end{array}$

146

10

10

L 8

L $\quad 32 / 10$

$-36 \quad 21 \quad 42$

4.05

36

$\begin{array}{llll}-9 & 57 & 3 & 3.83\end{array}$

53

$\begin{array}{llll}0 & 54 & -3 & 3.22\end{array}$

L 6

$\begin{array}{lll}-39 & 9 & 18\end{array}$

3.64

52

$\begin{array}{llll}-27 & 0 & 21 & 3.53\end{array}$

$\begin{array}{llll}-3 & -15 & 33 & 3.63\end{array}$

18

$-48 \quad-54$

33

3.62

52

$\begin{array}{lllll}-66 & -54 & 27 & 3.57\end{array}$

$\begin{array}{llll}-42 & -60 & 39 & 3.16\end{array}$

$\begin{array}{ll}\mathrm{R} & \mathrm{N} / \mathrm{A} \\ \mathrm{R} & 40 \\ \mathrm{~L} & 8 \\ \mathrm{R} & \mathrm{N} / \mathrm{A} \\ \mathrm{R} & 8\end{array}$

$30-9$

3

3.59

12

$\begin{array}{ll}48 & -57\end{array}$

45

3.47

8

$-18 \quad 27$

45

3.41

6

1899

3.38

10

33

24

48

3.35

21

$\begin{array}{llll}21 & 21 & 48 & 3.28\end{array}$

Regions significant at an uncorrected $p<.001$ with an extent $>5$ for the remembered-high versus forgotten covariate.

A maximum of 3 local maxima at least $8 \mathrm{~mm}$ apart are displayed per region of activation.

$\mathrm{BA}=$ Brodmann area (approximate).

frontal activations, suggesting that lowered activity in the parahippocampus was associated with robust activity in inferior frontal regions. This pattern bolsters an interpretation of compensatory engagement of frontal structures, particularly given that the correlations are positive in young adults. Second, additional correlations indicate that activation in inferior frontal regions unique to remembered items was correlated with left middle frontal activations in old but not young adults, again a finding supportive of a compensatory role for the middle frontal areas, working in conjunction with inferior frontal regions. The compensatory argument would be complete if a negative correlation existed between parahippocampal regions to middle frontal areas in only older adults. Although these correlations are negative, they do not reach significance. Nevertheless, these same correlations approach zero in young adults, so the pattern supports the hypothesized relationships.

A final finding that converges with past studies (Morcom et al., 2003) is evidence for subsequent forgetting effects (Otten \& Rugg, 2001; Wagner \& Davachi, 2001) in younger more so than in older adults. These frontopolar 
Table 4. Correlations as a Function of Age

Young

Old

Parahippocampal and Inferior Frontal Correlations

Left parahippocampal-

.42

$-.65 *$

Left inferior frontal

Left parahippocampal-

Right inferior frontal

Right parahippocampal-

Left inferior frontal

Right parahippocampal-

Right inferior frontal

\section{Middle Frontal and Inferior Frontal Correlations}

Left middle frontal.28

Left inferior frontal

Left middle frontal-

$$
-.32
$$

Right inferior frontal

Right middle frontal-

Left inferior frontal

Right middle frontal-

Right inferior frontal

.34

Middle Frontal and Parabippocampal Correlations

Left middle frontal-

Left parahippocampal

Left middle frontal-

Right parahippocampal

Right middle frontalLeft parahippocampal

Right middle frontal-

Right parahippocampal

*Correlation is significant at the .05 level (two-tailed).

effects were not limited to BA 10 as reported in earlier studies, but extended into the anterior cingulate region. The anterior cingulate has been linked to error detection and conflict resolution (Gehring \& Knight, 2000; Carter et al., 1998). Perhaps the anterior cingulate was engaged due to the large number of highly similar pictures studied at encoding, requiring disambiguation. In this paradigm, such a process should be relevant at encoding due to the inclusion of semantically similar scenes, which was necessary to create a large and difficult stimulus set. There is evidence that the elderly rely more on gist-based processing, rather than encoding many specific perceptual details (Koutstaal, Schacter, Galluccio, \& Stofer, 1999), so increased anterior cingulate activations may reflect young adults' increased sensitivity to the similarities and fine distinctions among the pictures. This explanation is consistent with Wagner and Davachi's (2001) suggestion that subsequent forgetting effects could reflect the failure of encoding pro- cesses to generate a unique trace for each item, leading to interference and forgetting.

In closing, the present findings point to increased frontal activation in older adults unique to remembered items, presenting a strong case for frontal activations as compensatory for other systemic declines, as proposed by Reuter-Lorenz (2002), Cabeza (2002), Reuter-Lorenz, Marshuetz, et al. (2001), and Grady, Maisog, et al. (1994). In addition, there is evidence that these frontal activations may specifically compensate for the decreased engagement of medial-temporal structures by older adults. Encoding of complex scenes places high demands on both frontal and medial-temporal structures and therefore represents an ideal paradigm for understanding connectivity and compensatory activations in these areas.

\section{METHODS}

\section{Participant Demographics}

A total of 14 young adults ( 7 men) from the University of Michigan and Ann Arbor community (average age = 21.0) and 13 community-dwelling elderly adults ( 7 men) from the Ann Arbor area (average age $=70.0$ ) participated in the study. All subjects were healthy and screened for right-handedness, contraindications for scanning, medications affecting blood flow or brain function, and psychiatric, neuropsychological, or structural abnormalities (including depression, dementia, and stroke). Two additional elderly adults completed the study but were excluded due to missing data or scanner malfunction. Between the two samples, there were no significant age differences on measures of years of education (young $=15.0$; elderly $=15.1$ ) or scores on the Mini-Mental State Exam (MMSE; Folstein, Folstein, \& McHugh, 1975) (young $=29.3$; elderly $=28.6$ ). All participants scored at least a 26 on the MMSE. Complete demographics information is presented in Table 5.

\section{Procedure}

All participants underwent two testing sessions. The first session consisted of neuropsychological testing and practice on a seemingly unrelated encoding task to familiarize them with the response requirements of the study. Functional imaging took place in the second session. Participants received US $\$ 75$ for participating, plus reimbursement for travel mileage. The University of Michigan Medical School Institutional Review Board approved the study protocol.

\section{Practice Session}

During the practice session, participants completed a neuropsychological battery (described below) in addi- 

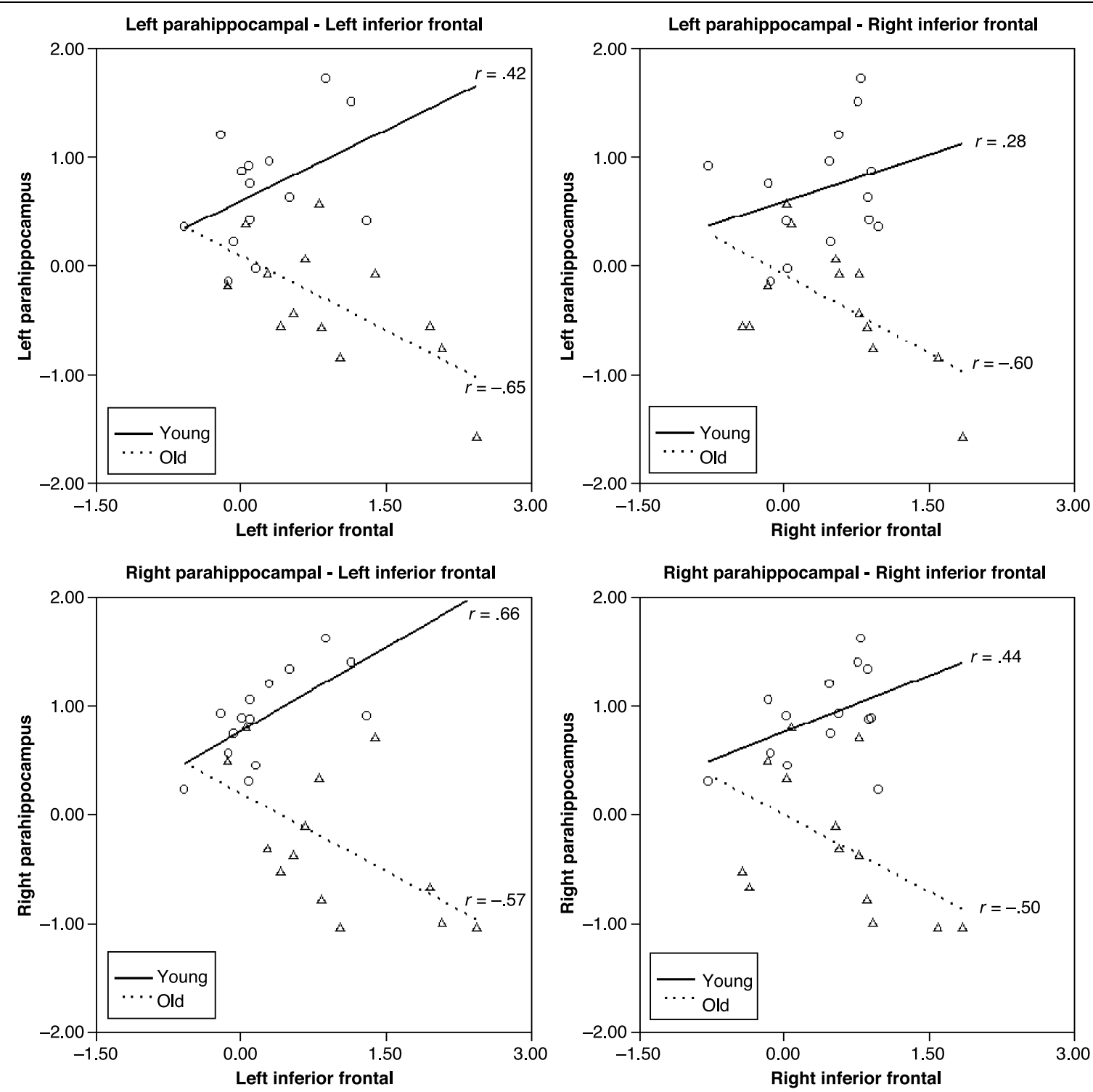

Figure 4. Scatterplots displaying differences in the correlations between parahippocampal and inferior frontal activations as a function of age. Each point represents the beta values for the difference between the remembered and forgotten estimates in the regions noted for a single participant. The linear regression functions are shown separately for younger and older adults and illustrate negative trends for older adults and more positive trends for younger adults.

tion to a word memory test. This test provided them the same response mappings that they would use to respond to the picture memory test in the scanner. Oral and written instructions emphasized speed and accuracy equally, however, participants were not told that this was practice for the fMRI task. This session lasted approximately $1 \mathrm{hr}$ and participants gave full written consent prior to participation.

\section{Neuropsychological Assessment}

In order to compare young and old samples on basic measures of cognitive ability, participants completed demographics and health questionnaires in addition to tasks to assess speed of processing and fluid and crystallized intelligence: Forward and Backward Digit Span (from the Wechsler Adult Intelligence Scale-III; Wechsler, 1997a), Letter-Number Sequencing (Wechsler Memory Scale-III; Wechsler, 1997b), Digit Comparison (adapted from the Letter Comparison Task of Salthouse \& Babcock, 1991), and Shipley Vocabulary (from the Shipley Institute of Living Scale; Shipley, 1986). Young participants performed significantly better than older participants on all of the measures with the exception of Shipley Vocabulary, on which the reverse was true (although nonsignificantly so). Results are presented in Table 5. Scores for the Digit Span represent the number of correct trials. In addition, there were no group differ- 
Table 5. Participant Characteristics, Demographics, and Neuropsychological Assessment (Means and Standard deviations)

\begin{tabular}{lccc}
\hline & Young & Old & Sig $(p)$ \\
\hline Age & $21.00(2.00)$ & $70.00(3.44)$ & .00 \\
Years of education & $14.96(1.67)$ & $15.12(2.33)$ & .85 \\
Mini-Mental State Exam & $29.29(1.07)$ & $28.62(1.33)$ & .16 \\
Shipley Vocabulary Task & $32.71(3.15)$ & $34.62(3.95)$ & .18 \\
Digit Comparison & $26.10(2.92)$ & $19.23(2.84)$ & .00 \\
Digit Span-forward & $12.43(2.38)$ & $10.54(1.98)$ & .04 \\
Digit Span-backward & $9.71(2.68)$ & $7.15(2.08)$ & .01 \\
Letter-number sequencing & $13.36(2.90)$ & $10.08(2.69)$ & .01 \\
\hline
\end{tabular}

ences on a 5-point scale in self-reported health in comparison to peers (young $=3.9$; elderly $=3.8$ where a rating of 4 denotes "above average") or health satisfaction (young $=4.0$; elderly $=3.9$ where a rating of 4 corresponds to "somewhat satisfied").

\section{fMRI Session}

The fMRI session lasted under $2 \mathrm{hr}$ and included 9 runs, each $6 \mathrm{~min}$ and $38 \mathrm{sec}$ in length. The first three runs consisted of picture encoding trials, whereas the final six consisted of picture recognition trials and are not included in the present analyses. Each run contained 97 events (either picture or baseline trials), each lasting exactly $4 \mathrm{sec}$ and presented in a pseudorandom ordering. Picture trials constituted two-thirds of the events per run, whereas the remaining one-third of the events were baselines. The task was presented using E-Prime software (Psychology Software Tools, Pittsburgh, PA) and the IFIS 9.0 system (MRI Devices, Waukesha, WI).

\section{Experimental Cognitive Task}

Participants incidentally encoded a total of 194 color photographs of outdoor scenes (see Figure 1) interspersed with 97 baseline trials consisting of a cross-hair fixation. Pictures of outdoor scenes were selected from commercially available CDs of full-color photographs. As each picture was presented, participants made a button press with their middle finger or thumb to assess whether or not water was present in the picture. After a 10-min delay, participants completed a recognition test on these 194 targets as well as 194 similar lures, interspersed with 194 baselines. They evaluated the pictures by pressing a button to indicate whether or not they remembered the pictures: "yes with high confidence," "yes with low confidence," or "no, did not study." Memory performance is shown in Table 1.

\section{Image Acquisition and Analysis}

\section{Acquisition}

All subjects participated in 9 fMRI runs (3.0 T GE LX gradient-echo spiral, $\mathrm{TR}=2000 \mathrm{msec}, \mathrm{TE}=25 \mathrm{msec}$, $\mathrm{FA}=80, \mathrm{FOV}=20 \mathrm{~cm}$ ) while performing the cognitive tasks in a full-body GE scanner (General Electric, Milwaukee, WI) outfitted with a standard head coil. Functional scans were $4 \mathrm{~mm}$ thick with an effective matrix of $64^{2}(3.125 \times 3.125 \mathrm{~mm})$, acquired at 32 contiguous locations approximately parallel to the anterior commissure -posterior commissure line. These slices covered the extent of the cortex and the cerebellum. Structural T1 images, co-planar with the T2* images, as well as highresolution 3D-SPGR $(0.9375 \mathrm{~mm}$ in-plane resolution, $1.5 \mathrm{~mm}$ thick slice) were collected during the session.

\section{Random-effects Analysis}

Image analysis. fMRI data were slice time corrected using an 8-point Hanning windowed sinc interpolation implemented in $\mathrm{C}++$. Intrasubject motion correction was performed using AIR 3.08 (Woods, Cherry, \& Mazziotta, 1992). SPM99 (Wellcome Department of Cognitive Neurology, London, UK) was used for transformation of image sets into a common stereotactic system (MNI), resampling images to $3-\mathrm{mm}^{3}$ voxels. Images were smoothed with a 6-mm gaussian kernel.

Statistical analysis was performed with SPM99. Individual analysis was conducted implementing a model of high confidence remembered items, low confidence remembered items, forgotten items, no response, and baseline. Contrast images were first calculated as the difference in the beta images: remembered-high confidence (hits- "yes-high confidence" responses) minus forgotten items (misses- "no" responses). Subsequently, these images were smoothed with an 8-mm gaussian kernel and tested in both a single-sample and a two-sample (between age groups) random-effects group analyses (Holmes \& Friston, 1998). For all comparisons, a threshold of $p<.001$ (uncorrected for multiple comparisons) and a spatial extent $>5$ voxels were used.

Laterality analysis. We probed the inferior frontal activations for laterality differences with age by creating ROIs. For the analysis of the regions of activation common to both younger and older adults, spheres with a $10-\mathrm{mm}$ radius (constrained by the boundaries of the activation) were created around the center of mass for both the left $(-51,36,6)$ and right $(48,36,15)$ inferior frontal functional activations. Beta values were then extracted for each participant for the size of the effect in each ROI. Using the scaled formula $\left[(\mathrm{L}-\mathrm{R}) / \sqrt{ }\left(\mathrm{L}^{2}+\right.\right.$ $\mathrm{R}^{2}$ )] as presented in Morcom et al. (2003), we computed a laterality index for each participant and subjected those to an independent sample $t$ test with age group as the 
between-subjects variable. For the analysis of laterality in the middle frontal regions more activated by older adults, ROIs were created and analyzed in the same manner, centered around $(-36,21,42)$ and $(33,24,48)$. The coordinates $(-21,-51,-3)$ and $(24,-48,-3)$ were used for the parahippocampal laterality analysis for regions activated more by young than old. Pearson's correlations were computed using these extracted effect sizes for parahippocampal and frontal regions.

\section{Acknowledgments}

Research was conducted in partial fulfillment of the first author's PhD requirements. Research was supported by the National Institute on Aging Neuroscience and Neuropsychology Program Grant R01AGO6265-15. We thank Julie Hautamaki for her experimental assistance, the University of Michigan Functional Imaging Center for their support, and Thad Polk, Carolyn Yoon, and two anonymous reviewers for their helpful comments.

Reprint requests should be sent to Angela Hall Gutchess, Department of Psychology, University of Michigan, 525 East University, Ann Arbor, MI 48109, or via e-mail: anghall@ umich.edu.

The data reported in this experiment have been deposited in the fMRI Data Center (www.fmridc.org). The accession number is 2-2004-115WA.

\section{REFERENCES}

Bäckman, L., Almkvist, O., Andersson, J., Nordberg, A., Windblad, B., Reineck, R., \& Långström, B. (1997). Brain activation in young and older adults during implicit and explicit retrieval. Journal of Cognitive Neuroscience, 9, 378-391.

Brewer, J. B., Zhao, Z., Desmond, J. E., Glover, G. H., \& Gabrieli, J. D. E. (1998). Making memories: Brain activity that predicts how well visual experience will be remembered. Science, 281, 1185-1187.

Cabeza, R. (2002). Hemispheric asymmetry reduction in older adults: The HAROLD model. Psychology and Aging, 17, 85-100.

Cabeza, R., Anderson, N. D., Locantore, J. K., \& McIntosh, A. R. (2002). Aging gracefully: Compensatory brain activity in high-performing older adults. Neuroimage, 17, 1394-1402.

Cabeza, R., Daselaar, S. M., Dolcos, F., Prince, S. E., Budde, M., \& Nyberg, L. (2004). Task-independent and task-specific age effects on brain activity during working memory, visual attention, and episodic retrieval. Cerebral Cortex, 14, 364-375.

Cabeza, R., Grady, C. L., Nyberg, L., McIntosh, A. R., Tulving, E., Kapur, S., Jennings, J. M., Houle, S., \& Craik, F. I. M. (1997). Age-related differences in neural activity during memory encoding and retrieval: A positron emission tomography study. Journal of Neuroscience. 17. 391-400.

Cabeza, R., Locantore, J. K., \& Anderson, N. D. (2003). Lateralization of prefrontal activity during episodic memory retrieval: Evidence for the production-monitoring hypothesis. Lournal of Cognitive Neuroscience, 15. 249-259.

Carter, C. S., Braver, T. S., Barch, D. M., Botvinick, M. M., Noll, D., \& Cohen, J. D. (1998). Anterior cingulate cortex, error detection, and the online monitoring of performance. Science, 280, 747-749.
Cohen, N. J., Ryan, J., Hunt, C., Romine, L., Wszalek, T., \& Nash, C. (1999). Hippocampal system and declarative (relational) memory: Summarizing the data from functional neuroimaging studies. Hippocampus, 9, 83-98.

Daselaar, S. M., Veltman, D. J., Rombouts, S. A., Lazeron, R. H., Raaijmakers, J. G., \& Jonker, C. (2003). Neuroanatomical correlates of episodic encoding and retrieval in young and elderly subjects. Brain, 126, 43-56.

Daselaar, S. M., Veltman, D. J., Rombouts, S. A., Raaijmakers, J. G., \& Jonker, C. (2003). Deep processing activates the medal temporal lobe in young but not elderly adults. Neurobiology of Aging, 24, 1005-1011.

Davachi, L., Mitchell, J. P., \& Wagner, A. D. (2003). Multiple routes to memory: Distinct medial temporal lobe processes build item and source memories. Proceedings of the National Academy of Sciences, U.S.A., 100, 2157-2162.

Folstein, M. F., Folstein, S. E., \& McHugh, P. R. (1975). Mini-mental state: A practical method for grading the cognitive state of patients for the clinician. Journal of Psychiatric Research, 12, 189-198.

Gehring, W. J., \& Knight, R. T. (2000). Prefrontal-cingulate interactions in action monitoring. Nature Neuroscience, 3, 516-520.

Golby, A. J., Poldrack, R. A., Brewer, J. B., Spencer, D., Desmond, J. E., Aron, A. P., \& Gabrieli, J. D. E. (2001). Material-specific lateralization in the medial temporal lobe and prefrontal cortex during memory encoding. Brain, 124, 1841-1854.

Grady, C. L., Bernstein, L. J., Beig, S., \& Siegenthaler, A. L. (2002). The effects of encoding strategy on age-related changes in the functional neuroanatomy of face memory. Psychology and Aging. 17, 7-23.

Grady, C. L., Maisog, J. M., Horwitz, B., Ungerleider, L. G., Mentis, M. J., Salerno, J. A., Pietrini, P., Wagner, E., \& Haxby, J. V. (1994). Age-related changes in cortical blood flow activation during visual processing of faces and location. Journal of Neuroscience, 14, 1450-1462.

Grady, C. L., McIntosh, A. R., \& Craik, F. I. M. (2003). Age-related differences in the functional connectivity of the hippocampus during memory encoding. Hippocampus, 13, 572-586.

Grady, C. L., McIntosh, A. R., Horwitz, B., Maisog, J. M., Ungerleider, L. G., Mentis, M. J., Pietrini, P., Schapiro, M. B., \& Haxby, J. V. (1995). Age-related reductions in human recognition memory due to impaired encoding. Science, 269, 218-221.

Grady, C. L., McIntosh, A. R., Rajah, M. N., Beig, S., \& Craik, F. I. M. (1999). The effects of age on the neural correlates of episodic encoding. Cerebral Cortex, 9, 805-814.

Grady, C. L., McIntosh, A. R., Rajah, M. N., \& Craik, F. I. M. (1998). Neural correlates of the episodic encoding of pictures and words. Proceedings of the National Academy of Sciences, U.S.A., 95, 2703-2708.

Hedden, T. \& Gabrieli, J. D. E. (2004). Insights into the aging mind: A view from cognitive neuroscience. Nature Reviews Neuroscience, 5, 87-96.

Henke, K., Buck, A., Weber, B., \& Wieser, H. G. (1997). Human hippocampus establishes associations in memory. Hippocampus. 7. 249-256.

Henke, K., Weber, B., Kneifel, S., Wieser, H. G., \& Buck, A. (1999). Human hippocampus associates information in memory. Proceedings of the National Academy of Sciences, U.S.A., 96, 5884-5889.

Holmes, A. P., \& Friston, K. J. (1998). Generalisability, random effects and population inference. Neuroimage, 7, S754.

Jennings, J. M., \& Jacoby, L. L. (1993). Automatic versus intentional uses of memory: Aging, attention, and control. Psychology and Aging, 8, 283-293. 
Jennings, J. M., \& Jacoby, L. L. (1997). An opposition procedure for detecting age-related deficits in recollection: Telling effects of repetition. Psychology and Aging, 12, 352-361.

Kelley, W. M., Miezin, F. M., McDermott, K. B., Buckner, R. L., Raichle, M. E., Cohen, N. J., Ollinger, J. M., Akbudak, E., Conturo, T. E., Snyder, A. Z., \& Petersen, S. E. (1998). Hemispheric specialization in human dorsal frontal cortex and medial temporal lobe for verbal and nonverbal memory encoding. Neuron, 20, 927-936.

Kirchhoff, B. A., Wagner, A. D., Maril, A., \& Stern, C. E. (2000). Prefrontal-temporal circuitry for episodic encoding and subsequent memory. Journal of Neuroscience, 20, 6173-6180.

Koutstaal, W., Schacter, D. L., Galluccio, L., \& Stofer, K. A. (1999). Reducing gist-based false recognition in older adults: Encoding and retrieval manipulations. Psychology and Aging, 14, 220-237.

Logan, J. M., Sanders, A. L., Snyder, A., Morris, J. C., \& Buckner, R. L. (2002). Under-recruitment and non-selective recruitment: Dissociable neural mechanisms associated with aging. Neuron, 33, 827-840.

Lustig, C., Snyder, A. Z., Bhakta, M., O’Brien, K., McAvoy, M., Raichle, M. E., Morris, J. C., \& Buckner, R. L. (2003). Functional deactivations: Change with age and dementia of the Alzheimer type. Proceedings of the National Academy of Sciences, U.S.A., 100, 14504-14509.

Madden, D. J., Turkington, T. G., Provenzale, J. M., Denny, L. L., Hawk, T. C., Gottlob, L. R., \& Coleman, R. E. (1999). Adult age differences in functional neuroanatomy of verbal recognition memory. Human Brain Mapping, 7, 115-135.

Maguire, E. A., \& Frith, C. D. (2003). Aging affects the engagement of the hippocampus during autobiographical memory retrieval. Brain, 126, 1511-1523.

McIntosh, A. R., Sekuler, A. B., Penpeci, C., Rajah, M. N., Grady, C. L., Sekuler, R., \& Bennett, P. J. (1999). Recruitment of unique neural systems to support visual memory in normal aging. Current Biology, 9, 1275-1278.

Miller, E. K., \& Cohen, J. D. (2001). An integrative theory of prefrontal cortex function. Annual Review of Neuroscience, 24, 167-202.

Morcom, A. M., Good, C. D., Frackowiak, R. S., \& Rugg, M. D. (2003). Age effects on the neural correlates of successful memory encoding. Brain, 126, 213-229.

Moscovitch, M., \& Winocur, G. (1992). The neuropsychology of memory and aging. In F. I. M. Craik \& T. A. Salthouse (Eds.), The handbook of aging and cognition (pp. 315-372). Hillsdale, NJ: Erlbaum.

Otten, L. J., \& Rugg, M. D. (2001). When more means less: Neural activity related to unsuccessful memory encoding. Current Biologv, 11, 1528-1530.

Paivio, A., \& Csapo, K. (1973). Picture superiority in free recall: Imagery or dual coding? Cognitive Psychology, 5, 176-206.

Park, D. C., \& Gutchess, A. H. (in press). Long-term memory and aging: A cognitive neuroscience perspective. In $\mathrm{R}$. Cabeza, L. Nyberg, \& D. C. Park (Eds.), Cognitive neuroscience of aging: Linking cognitive and cerebral aging. New York: Oxford Press.

Park, D. C., Puglisi, J. T., \& Smith, A. D. (1986). Memory for pictures: Does an age-related decline exist? Psychology and Aging, 1, 11-17.

Park, D. C., Puglisi, J. T., Smith, A. D., \& Dudley, W. N. (1987). Cue utilization and encoding specificity in picture recognition by older adults. Journal of Gerontology, 42, 423-425.

Park, D. C., Welsh, R. C., Marshuetz, C., Gutchess, A. H., Mikels, J., Polk, T. A., Noll, D. C., \& Taylor, S. F. (2003). Age differences in frontal and medial temporal activations associated with the maintenance of complex pictures in working memory. Journal of Cognitive Neuroscience, 15, 1122-1134.

Raz, N. (2000). Aging of the brain and its impact on cognitive performance: Integration of structural and functional findings. In F. I. M. Craik \& T. A. Salthouse (Eds.), Handbook of aging and cognition (pp. 1-90). Mahwah, NJ: Erlbaum.

Reber, P. J., Wong, E. C., \& Buxton, R. B. (2002). Encoding activity in the medial temporal lobe examined with anatomically constrained fMRI analysis. Hippocampus, 12 , $363-376$.

Reuter-Lorenz, P. A. (2002). New visions of the aging mind and brain. Trends in Cognitive Sciences, 6, 394-400.

Reuter-Lorenz, P. A., Jonides, J., Smith, E., Hartley, A., Miller, A., Marshuetz, C., \& Koeppe, R. (2000). Age differences in the frontal lateralization of verbal and spatial working memory revealed by PET. Journal of Cognitive Neuroscience, 12, 174-187.

Reuter-Lorenz, P. A., Marshuetz, C., Jonides, J., Smith, E. E., Hartley, A., \& Koeppe, R. (2001). Neurocognitive ageing of storage and executive processes. European Journal of Cognitive Psychology, 13, 257-278.

Rosen, A. C., Prull, M. W., O'Hara, R., Race, E. A., Desmond, J. E., Glover, G. H., Yesavage, J. A., \& Gabrieli, J. D. E. (2002). Variable effects of aging on frontal lobe contributions to memory. NeuroReport, 13, 2425-2428.

Rypma, B., \& D'Esposito, M. (2000). Isolating the neural mechanisms of age-related changes in human working memory. Nature Neuroscience, 3, 509-515.

Salthouse, T. A., \& Babcock, R. L. (1991). Decomposing adult age differences in working memory. Developmental Psychology. 27. 763-776.

Shipley, W. C. (1986). Shipley Institute of Living Scale. Los Angeles: Western Psychological Services.

Smith, A. D., Park, D. C., Cherry, K., \& Berkovsky, K. (1990). Age differences in memory for concrete and abstract pictures. Journal of Gerontology, 35, 205-209.

Snodgrass, J. G., \& Corwin, J. (1988). Pragmatics of measuring recognition memory: Applications to dementia and amnesia. Journal of Experimental Psychology: General, 117, 34-50.

Stebbins, G. T., Carrillo, M. C., Dorfman, J., Dirksen, C., Desmond, J. E., Turner, D. A., Bennett, D. A., Wilson, R. S., Glover, G., \& Gabrieli, J. D. E. (2002). Aging effects on memory encoding in the frontal lobes. Psychology and Aging, 17, 44-55.

Stern, C. E., Corkin, S., Gonzalez, R. G., Guimaraes, A. R., Baker, J. R., Jennings, P. J., Carr, C. A., Sugiura, R. M., Vedantham, V., \& Rosen, B. R. (1996). The hippocampal formation participates in novel picture encoding: Evidence from functional magnetic resonance imaging. Proceedings of the National Academy of Sciences, U.S.A., 93, 8660-8665.

Tulving, E. (2002). Episodic memory: From mind to brain. Annual Review of Psychology, 53, 1-25.

Wagner, A. D., \& Davachi, L. (2001). Cognitive neuroscience: Forgetting of things past. Current Biology, 11, R964-R967.

Wagner, A. D., Schacter, D. L., Rotte, M., Koutstaal, W., Maril, A., Dale, A. M., Rosen, B. R., \& Buckner, R. L. (1998). Building memories: Remembering and forgetting of verbal experiences as predicted by brain activity. Science, 281. 1188-1191.

Wechsler, D. (1997a). Wechsler Adult Intelligence Scale (3rd ed.). San Antonio, TX: The Psychological Corporation. Wechsler, D. (1997b). Wechsler Memory Scale (3rd ed.). San Antonio, TX: The Psychological Corporation.

Woods R. P., Cherry S. R., \& Mazziotta J. C. (1992). Rapid automated algorithm for aligning and reslicing PET images. Journal of Computer Assisted Tomography, 16, 620-633. 